\title{
Una mirada a la productividad laboral para las pymes de confecciones
}

\section{A look at labor productivity for SMEs in the clothing industry}

\author{
Ludym Jaimes-Carrillo \\ Ph. D. (c). Ingeniería \\ Universidad Nacional de Colombia \\ Medellín, Colombia \\ ljaimesc@unal.edu.co
}

\author{
Miguel David Rojas-López \\ Ph. D. Ingeniería \\ Universidad Nacional de Colombia. \\ Medellín, Colombia \\ mdrojası@unal.edu.co
}

Resumen- El interés por la productividad, específicamente por la productividad laboral, está incrementándose, la motivación es encontrar los aspectos influyentes para mejorarla. El objetivo es evidenciar la necesidad de un modelo para aumentar la productividad laboral en pymes del sector confecciones. Se presenta una revisión de literatura, identificando aportes e información relevante para la construcción futura de dicho modelo, construido para el contexto del área Metropolitana de Bucaramanga, Santander. La investigación aborda la productividad laboral desde los enfoques de la economía, la administración y la ingeniería. Dicha productividad es primordial, más aún para las pymes, dadas sus necesidades de orientación y su impacto en los ingresos y el empleo nacional. A lo largo de la historia de Bucaramanga el sector confecciones es considerado como estratégico, por esto es relevante gestionar su productividad laboral para impactar el desarrollo local. Este escrito resalta los principales aspectos para la futura construcción del modelo mencionado.

Palabras clave- Productividad laboral, pyme, sector confecciones.

Abstract- The interest for the productivity, and specifically by labor productivity has increased, the motivation lies in finding the most influential aspects to improve organizational productivity. The aim is to highlight the need for a model to increase labor productivity in SMEs in the clothing industry. This paper is looking for relevant information to construct that model; it will build to the context of the metropolitan area of Bucaramanga, Santander. The research raises labor productivity from the approaches of the economy, administration and engineering. This productivity is vital for organizations; even more for SMEs has given their need for orientation and its impact on income and the employment at the national level. Throughout the history of Bucaramanga the clothing industry has been regarded as strategic, for this reason is relevant manage their labor productivity to impact local development. This paper highlights the main aspects to consider for the future construction of the model mentioned.

Keywords- clothing industry, labor productivity, pyme.

\section{INTRODUCCIÓN}

La productividad día por día es un término más cotidiano, a nivel empresarial, sectorial, regional y nacional. Su relevancia es resumida por Porter [1] al afirmar "La productividad es el determinante fundamental del nivel de vida de una nación a largo plazo... El nivel de vida de una nación depende de la capacidad de sus empresas para lograr altos niveles de productividad y para aumentar esta a lo largo del tiempo" (p. 168).

El tema central de este escrito es la productividad del factor humano, denominada productividad laboral (PL), dado su impacto en el desempeño empresarial. El interés por estudiar la productividad y específicamente la PL radica en encontrar los aspectos influyentes en la mejora de la productividad organizacional.

Se plantean a lo largo del tiempo diversas metodologías de medición de la PL. Considerándola como la relación entre las salidas del proceso productivo y las entradas de capital de trabajo; sin embargo, dependiendo de la mirada desde la cual se estudie y las motivaciones de los investigadores, existen diversos planteamientos sobre el tema. 
A pesar de la extensa literatura sobre productividad, y en menor grado sobre $\mathrm{PL}$, las pequeñas y medianas empresas - pymes- del sector confecciones del área Metropolitana de Bucaramanga (AMB) carecen de una metodología para gestionarla, que se adecue a sus recursos, necesidades y expectativas.

Esta problemática motiva la investigación sobre la PL para pymes de confecciones del AMB, en busca de establecer un modelo. Este escrito presenta elementos sobre los antecedentes de la PL en el país, la región y el sector, así como algunas razones que justifican el diseño de dicho modelo para las pymes del sector confecciones del AMB.

Considerando como área de investigación la productividad, y puntualmente la PL se puede resaltar la "necesidad de dar un nuevo impulso a la empresa en la búsqueda de la competitividad y de desplegar en el trabajo una dimensión humana que hasta ahora, para la mayoría, ha estado excluida" [2]. Aunque este fragmento se enfoca en la competitividad, tiene una relación con el área planteada, ya que "la verdadera competitividad se mide con la productividad" [1].

De acuerdo con la revisión bibliográfica realizada, a la fecha no se cuenta con un modelo que explique la PL de las pymes, específicamente en el sector confecciones, lo cual restringe la visión y acción de las organizaciones en el manejo de esta. La productividad es el determinante primordial de la calidad de vida de un país [1]. De manera que al identificar las variables que explican la $\mathrm{PL}$ y las relaciones entre ellas, se abren caminos para su gestión en las organizaciones, aportando a la competitividad empresarial, al desarrollo regional y al aumento de la calidad de vida en general.

El alcance de este escrito es evidenciar la necesidad de contar con un modelo para aumentar la $\mathrm{PL}$ en las pymes del sector confecciones. Para lograrlo, se realiza una revisión de estudios relevantes sobre el tema, resaltando la dificultad de adopción de los planteamientos por parte de las pymes del sector confecciones. Los resultados de las investigaciones sobre PL en ocasiones no son válidas para las pymes debido a las particularidades en la gestión de las mismas, como el hecho de contar un solo gerente -y propietario, en múltiples ocasiones- a cargo de todas las áreas funcionales [3].
Muñoz [4] en su estudio del clúster de confección textil y moda de Medellín, desarrollado en 17 empresas, encontró que la cultura de no medir la productividad se debe en gran medida a la existencia de una gerencia poco capacitada en temas relacionados con la administración y la productividad.

De otro lado, se resalta la trascendencia del sector confecciones a lo largo de la historia a nivel nacional, y en el área local de Bucaramanga. El estudio se centró en el AMB considerando que de 1317 empresas de Santander registradas ante la Cámara de Comercio dedicadas a la confección de prendas de vestir, 1218 están ubicadas en el AMB, es decir, el 92.5\%, según información correspondiente al primer trimestre de 2014 [5].

El sector confecciones constantemente se perfila como foco estratégico departamental en los proyectos o programas para apalancar la competitividad de Santander, evidenciando la necesidad de abordar su gestión de la PL.

Reconociendo la relevancia del sector, se da paso a justificar la necesidad de estudiar la PL para pymes de confecciones. Dada la falencia de planteamientos teóricos sobre la gestión de la $\mathrm{PL}$ en pymes, lo cual es aún más relevante en el sector de interés, ya que la industria textil y principalmente la de confecciones se caracteriza por procesos con uso intensivo de mano de obra, principalmente no calificada [6]. Los costos laborales en Colombia son altos [7] lo cual estimula el interés por su control, así como por la PL considerando su estrecha relación.

La estructura del artículo cuenta con siete partes, la primera corresponde a esta introducción, la segunda presenta los antecedentes del problema; a continuación el objetivo y la metodología del trabajo realizado; la siguiente, muestra los resultados de la revisión de la literatura sobre conceptos y estudios de productividad y específicamente de PL; la sexta sección es la discusión de los hallazgos de la revisión bibliográfica, y la última, presenta las conclusiones.

\section{ANTECEDENTES DEL PROBLEMA}

Este aparte presenta desde el ámbito nacional, regional y local los antecedentes asociados con la forma como se ha trabajado la productividad y la PL, dando claridad sobre la situación problemáti- 
ca asociada a la carencia de un modelo de gestión de PL para pymes de confecciones.

\subsection{Desde el ámbito nacional}

La actual Política Nacional de Competitividad y Productividad [8] en su capítulo diagnóstico afirma: "Para mejorar su competitividad, Colombia debe enfrentar el reto que supone su baja productividad por hora trabajada. En términos promedio, la productividad relativa del país, ajustada por el poder de compra, es tan solo una quinta parte de la de Estados Unidos - EE. UU.". La Política de Desarrollo Productivo para Colombia [9], emitida en 2014, muestra cómo este comparativo con el paso del tiempo se mantiene con variaciones mínimas; se observa en la Fig. 1 la comparación de la PL relativa de Colombia frente e EE. UU. según datos de 2012.

La inclusión de la preocupación por la baja PL del país frente a la de EE. UU. en el 2005 en los aspectos diagnósticos de la Política de Productividad y Competitividad evidencia la estrecha relación entre estas, ante la presión por ser competitivos, la productividad se vuelve una condición necesaria, aunque no suficiente, para la anhelada competitividad de las organizaciones.

Fig. 1. PRODUCTIVIDAD LABORAL POR SECTORES - COMPARACIÓN ENTRE COLOMBIA Y ESTADOS UNIDOS, 2012

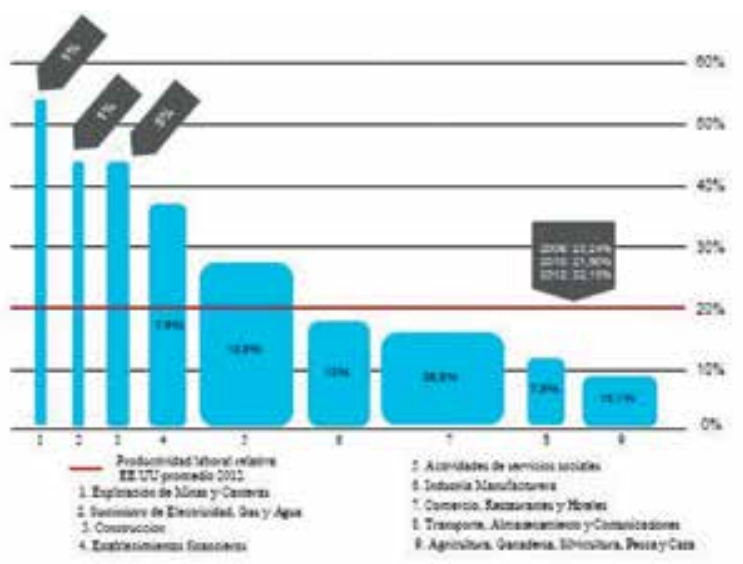

Fuente: Política de Desarrollo Productivo para Colombia [9].

La Fig. 1 muestra los diversos sectores organizados descendentemente según el nivel promedio de PL relativa de Colombia frente a EE. UU. en el 2012. El sector industrial se ubica ligeramente por debajo del promedio sectorial, también se puede observar la relevancia de la participación de la industria en el total de empleo generado a nivel nacional.

En la figura el 100\% como límite superior corresponde a la productividad de Estados Unidos, calculada como el valor agregado/número de empleados, es decir, como PL. Este valor incluye la productividad formal e informal de la economía americana.

Para el interés particular de este escrito, se puede ver la relevancia de la PL a nivel nacional en la inclusión permanente del tema, es así como año a año en los informes nacionales de competitividad se representa la evolución -o involución- de la PL desde el 2007 hasta la fecha.

El Informe Nacional de Competitividad 2012-2013 [10] plantea que el mercado laboral colombiano es percibido como poco competitivo en el contexto internacional debido a la falta de flexibilidad. La medición del Institute for Management Development [11] sitúa la PL nacional en la posición 38 entre 59 países. De otro lado, en el Informe Global de Competitividad del World Economic Forum [12], el país ocupó el lugar 88 entre 144 en el pilar "eficiencia del mercado laboral", conservando la misma posición del año anterior.

El Informe Nacional de Productividad 20132014 [13] menciona cuatro aspectos claves sobre el mercado laboral colombiano y su productividad:

i) Existe una estrecha relación positiva entre los niveles de formalidad y productividad; ii) la informalidad en Colombia varía de sector a sector; iii) existen grandes brechas de productividad intersectorial; y iv) los sectores donde se concentra la mayor cantidad de fuerza laboral son los más informales. Adicionalmente, hay evidencia de que en Colombia también hay amplias brechas de productividad en el interior de cada sector, donde en muchos sectores la gran mayoría de empresas tiene bajos niveles de productividad.

Según McMillan y Rodrik [14] las brechas en PL- intra e inter sectoriales caracterizan a los países en desarrollo, ya que evidencian la ineficiente asignación de recursos, por lo cual se disminuye la PL agregada de un país. 
El capítulo de mercado laboral y formalización del Informe Nacional de Competitividad 2014-2015 inicia enunciando: “El uso óptimo del factor trabajo es fundamental para el crecimiento económico y el desarrollo social de un país. El desempleo y la informalidad son reflejos de una ineficiente asignación de este factor, al tiempo que son causa y resultado de la baja productividad de un país" [15].

\subsection{Desde el ámbito sectorial}

El sector de textiles y confecciones es importante en Colombia, siendo un sector tradicional que tiene más de 100 años de experiencia en producción. Para ver la relevancia del sector confecciones a nivel nacional se puede considerar en primer lugar su inclusión en el estudio de competitividad de la economía colombiana desarrollada por Monitor Company a inicios de los noventa. El cual tenía como objetivo identificar cómo Colombia, a largo plazo, podía ampliar y mejorar la competitividad en una amplia gama de industrias. El estudio se centró en seis sectores, uno de ellos textiles y confecciones, el informe menciona que fue seleccionado por ser gran empleador, industria históricamente importante para la economía y con concentración regional [17], aspectos que a la fecha siguen siendo válidos.

De igual forma, en el 2008 el Gobierno nacional, a través del Ministerio de Comercio, Industria y Turismo creó el programa de transformación productiva (PTP), con el objetivo de mejorar la competitividad de diferentes sectores de la economía colombiana. Los sectores seleccionados se agruparon en dos categorías: nuevos o emergentes y más y mejor de lo bueno; en esta última se encontraba el sector textil y confecciones [18].

El informe de gestión del PTP 2012 [19] en los resultados por sector, plantea para el sistema moda que la principal brecha en la que se debe trabajar, es la gestión de la productividad. El "sistema moda", es un gran sector conformado por las industrias textil, confección, diseño y moda y cuero, calzado y marroquinería.

Este sector se caracteriza por ser gran empleador, en 2008 generó aproximadamente 600 mil empleos entre directos e indirectos, además, participa con el 10,3\% del PIB manufacturero y con el 1,6\% del total del PIB nacional [20]. Según datos del DANE, citados por Inexmoda la Cadena textil/confección en el año 2010 representó el $1.0 \%$ del Producto interno bruto nacional y el $10 \%$ del PIB manufacturero [21].

Algunos datos recientes del sector dejan ver su importancia a nivel nacional, el informe del PTP [19] enuncia: "Se estima que durante el 2012, las ventas del sector textil-confección, superaron los 5400 millones de dólares: un 46\% corresponde a textiles y un $54 \%$ a confecciones. La producción real del sector de textiles ha crecido a una tasa promedio anual de $2,5 \%$ en el periodo 2002 - 2012, mientras que el sector de confecciones aumentó su producción a un ritmo anual del 5,4\%".

\subsection{Desde el ámbito local}

Para resaltar la trascendencia del sector en Bucaramanga y su área metropolitana se presentan algunas iniciativas para el aumento de la competitividad de Santander comprendidas entre 1990 y 2005 , las cuales incluyen el sector confecciones como foco estratégico [22].

En la época de la apertura económica del país, la firma Araujo Ibarra y Asociados realizó en 1992 la priorización de los sectores productivos que deberían incorporarse a la oferta exportadora del departamento; en el sector de manufactura se eligieron confecciones, marroquinería, calzado y joyería. Este estudio recomendó para el sector de las confecciones la creación de una comercializadora, la diversificación de las líneas de exportación y la creación de un centro de información sobre moda [23].

La Gobernación de Santander y el Consejo Asesor Regional de Comercio Exterior en el 2001 plantearon la Estrategia de Competitividad de Santander [22]. Este proceso inició con un diagnóstico de las principales actividades empresariales del departamento, a partir de esta información se estableció la oferta exportable departamental en los sectores: avícola, artes gráficas, confecciones, cuero y calzado, joyería, palma y salud. Los líderes del proceso señalaron que dichos sectores en la época tenían alto impacto en la generación de empleo, y contaban con potencial exportador. 
En el 2002 la Corporación Metropolitana de Planeación y Desarrollo de Bucaramanga (Corplan) trabajó en el plan estratégico del AMB para el 2015 [24]. Este plan estableció seis líneas estratégicas: Ciudad integradora, incluyente y participativa; Ciudad educadora y cultural; Ciudad competitiva; Hábitat sustentable; Gestión pública eficiente y relacional y Metrópolis integrada regionalmente. En la línea de Ciudad competitiva se realizaron las propuestas sectoriales para apalancar el desarrollo regional, uno de los sectores seleccionados fue confecciones.

Más adelante, en el 2004 surge la Agenda Interna para la productividad y la competitividad definida como un "acuerdo de voluntades y decisiones entre la Nación, las entidades territoriales, el sector privado, los actores políticos y la sociedad civil sobre el conjunto de acciones estratégicas que el país debe realizar en el corto, mediano y largo plazo, para mejorar la productividad y competitividad de su aparato productivo" [25].

El proceso de construcción de la Agenda Interna tuvo tres ejes temáticos: el sectorial, el regional y el transversal. En las primeras etapas de avance de lo establecido en la agenda se elaboraron propuestas sectoriales en cada una de las direcciones técnicas del Departamento Nacional de Planeación; a nivel regional, en Santander, el sector confecciones hizo parte de los sectores estratégicos de trabajo para la productividad y competitividad del departamento [25].

Los estudios mencionados dan evidencia de la relevancia del sector confecciones como foco estratégico para la mejora de la competitividad a nivel nacional, de Santander y del AMB.

Santander y principalmente el AMB, es reconocida a nivel nacional como uno de los centros industriales de mayor importancia en la ropa infantil; según registros de 2008 de la Cámara de Comercio de Bucaramanga, en esta ciudad existen más de 110 empresas entre pymes y microempresas dedicadas a la producción de ropa infantil, las cuales generan más de 600 empleos directos en la zona, estas empresas cuentan con activos totales superiores a los $\$ 14000$ millones de pesos [20]. Esto hace que sea considerado como un sector estratégico en el Plan de Desarrollo del Departamento desde tiempo atrás, situación que se mantiene para el período actual, 2012-2015 [26].

\section{OBJETIVO}

Reconocer la importancia de la $\mathrm{PL}$, en particular para las pymes del sector de confecciones, para dar evidencia de la necesidad de diseñar un modelo de PL de acuerdo con las condiciones de sus procesos y el papel que juega en la economía local.

\section{METOdOLOGÍA}

La metodología seguida para este trabajo fue la revisión de literatura. Dicha revisión, se centró en artículos significativos a criterio del autor, de acuerdo con los siguientes pasos: i) Conceptualización de PL desde los enfoques de la economía, la administración y la ingeniería. ii) Identificación de aportes relevantes sobre relación de variables con la productividad o PL o modelos de las mismas, desde los tres enfoques mencionados.

\section{RESULTADOS}

Los resultados se presentan en dos secciones. En primer lugar, se incluye la conceptualización sobre PL desde los enfoques de la economía, la administración y la ingeniería. Finalmente, se enuncian algunos modelos y variables relacionadas con la productividad, desde los enfoques considerados.

\section{1 Conceptualización sobre PL}

Tradicionalmente la PL es estudiada desde la economía, la ingeniería y la administración, sin embargo, cada vez se encuentran más investigaciones desde disciplinas como: la psicología, la salud, la sociología, la antropología y la contabilidad, entre otras. La revisión de literatura realizada se centró en estudiar la PL desde los primeros enfoques mencionados. La tabla I presenta las propuestas conceptuales de algunos autores sobre la PL desde los tres enfoques señalados.

La tabla II recopila trabajos recientes sobre $\mathrm{PL}$ desde los tres enfoques de estudio, se presenta la generalidad de cada investigación y su principal aporte. Esta revisión se realizó seleccionando aquellos trabajos sobre PL con aportes relevantes para la futura construcción del modelo de PL para pymes del sector confecciones. 
TABLA

CONCEPTOS DE PRODUCTIVIDAD LABORAL

\begin{tabular}{|c|c|}
\hline AUTOR & DEFINICIÓN \\
\hline \multicolumn{2}{|r|}{ Enfoque económico } \\
\hline Adam Smith [27] & $\begin{array}{l}\text { "El producto anual de la tierra y del trabajo de la nación solo puede aumentarse por dos procedimientos: o } \\
\text { con un adelanto en las facultades productivas del trabajo útil que dentro de ellas se mantiene, o por algún } \\
\text { aumento en la cantidad de ese trabajo. El adelanto de las facultades productivas depende, ante todo, de los } \\
\text { progresos de las habilidades del operario, y en segundo término de los progresos de la maquinaria con que } \\
\text { se trabaja". }\end{array}$ \\
\hline $\begin{array}{l}\text { Organización Interna- } \\
\text { cional del Trabajo - } \\
\text { OIT- para América Lati- } \\
\text { na y el Caribe [28] }\end{array}$ & $\begin{array}{l}\text { La PL es un indicador clave para la medición del desarrollo, ya que relaciona aspectos productivos y socio- } \\
\text { laborales. En lo productivo, es el principal indicador de la brecha externa que separa a los países de América } \\
\text { Latina y el Caribe de las economías más desarrolladas... En el campo socio-laboral, no es casual que la PL, } \\
\text { medida como el PIB por persona ocupada, haya sido seleccionada como uno de los indicadores que ilustran } \\
\text { los avances y retrocesos de la meta 1B - "Lograr el empleo pleno y productivo y el trabajo decente para todos, } \\
\text { incluidos las y los jóvenes" - del primer objetivo del Milenio- "Erradicar la pobreza extrema y el hambre“. }\end{array}$ \\
\hline \multicolumn{2}{|r|}{ Enfoque de ingeniería } \\
\hline $\begin{array}{l}\text { Portal de productividad } \\
\text { laboral [29] }\end{array}$ & $\begin{array}{l}\text { La PL es el resultado de un sistema inteligente que permite a las personas en un centro de trabajo, optimizar } \\
\text { la aportación de todos los recursos materiales, financieros y tecnológicos que concurren en la empresa, para } \\
\text { producir bienes y servicios con el fin de promover la competitividad de la economía nacional, mejorar la sus- } \\
\text { tentabilidad de la empresa, así como de mantener y ampliar la planta productiva nacional e incrementar los } \\
\text { ingresos de los trabajadores. }\end{array}$ \\
\hline \multicolumn{2}{|r|}{ Enfoque administrativo } \\
\hline $\begin{array}{l}\text { Drucker [30]; England } \\
\text { [31] y Ericson [32] }\end{array}$ & $\begin{array}{l}\text { La productividad desde la administración es vista como una meta organizacional, en [30], [31], [32] lo inclu- } \\
\text { yen como uno de los objetivos por alcanzar en una empresa. }\end{array}$ \\
\hline Botero [33] & $\begin{array}{l}\text { La administración se centra en la reducción de costos, lo cual corresponde a la relación entre el concepto de } \\
\text { productividad tal y como los economistas lo han desarrollado y el concepto que en la práctica empresarial le } \\
\text { corresponde. }\end{array}$ \\
\hline
\end{tabular}

Fuente: autores.

TABLA II

ESTUDIOS DE PRODUCTIVIDAD LABORAL

\begin{tabular}{|c|c|c|}
\hline AUTOR & ESTUDIO & APORTE \\
\hline \multicolumn{3}{|c|}{ Enfoque económico } \\
\hline Dobija [34] & $\begin{array}{l}\text { Estudio de la PL como un factor esencial para estable- } \\
\text { cer el nivel salarial mínimo adecuado. }\end{array}$ & $\begin{array}{l}\text { El nivel y la equidad de los salarios mínimos legales están } \\
\text { condicionados por la PL. }\end{array}$ \\
\hline $\begin{array}{l}\text { Jedrzejczyk } \\
{[35]}\end{array}$ & $\begin{array}{l}\text { Verificación estadística de la PL como determinante } \\
\text { del comportamiento de la tasa de cambio. Estudiando } \\
\text { la PL como el cociente del PIB real sobre el costo de } \\
\text { la mano de obra y la tasa de cambio como el cociente } \\
\text { del salario por hora promedio. }\end{array}$ & $\begin{array}{l}\text { El valor del indicador PL es mayor en los países ricos y menor } \\
\text { en los países en desarrollo. }\end{array}$ \\
\hline Lisi [36] & $\begin{array}{l}\text { Estudio de la relación entre la flexibilidad del régimen } \\
\text { laboral, la productividad y el desempleo. }\end{array}$ & $\begin{array}{l}\text { El aumento en el corto plazo de la flexibilidad en el régimen } \\
\text { laboral no puede representar una solución definitiva al des- } \\
\text { empleo, ya que una solución estructural debe tener en cuen- } \\
\text { ta las necesidades de todos los sectores. }\end{array}$ \\
\hline $\begin{array}{l}\text { Sultana \& } \\
\text { Ktiroda [37] }\end{array}$ & $\begin{array}{l}\text { Investigaron los factores responsables de una alta } \\
\text { tasa de crecimiento de la PL del sector agrícola para } \\
\text { el período 1976-1993 en Taiwán. }\end{array}$ & $\begin{array}{l}\text { El efecto de sustitución total, debido a los cambios de pre- } \\
\text { cios de los factores y el cambio tecnológico sesgado fue } \\
\text { mayor al el efecto de la productividad total de los factores, } \\
\text { debido a las economías de escala y el progreso tecnológico. }\end{array}$ \\
\hline Olimpia [38] & $\begin{array}{l}\text { Explora la relación entre la productividad del trabajo } \\
\text { y las estimaciones del capital humano en los países } \\
\text { de la Unión Europea. La teoría del capital humano } \\
\text { explica el nivel de PL por nivel de educación de los } \\
\text { trabajadores. }\end{array}$ & $\begin{array}{l}\text { Se determinó que la productividad del trabajo está correla- } \\
\text { cionada fuertemente y de forma positiva con el stock de ca- } \\
\text { pital humano en las economías europeas. }\end{array}$ \\
\hline
\end{tabular}


Enfoque de ingeniería

\begin{tabular}{|c|c|c|}
\hline \multicolumn{3}{|c|}{ Enfoque de ingeniería } \\
\hline $\begin{array}{l}\text { Mawdesley y } \\
\text { Al-Jibouri [39] }\end{array}$ & $\begin{array}{l}\text { Desde la dinámica de sistemas -DS- exploró qué fac- } \\
\text { tores afectan la productividad en el sector construc- } \\
\text { ción del Reino Unido, cómo interactúan estos factores } \\
\text { y su importancia. }\end{array}$ & $\begin{array}{l}\text { Las cinco variables que favorecen la productividad en el sec- } \\
\text { tor construcción en Reino Unido son: inversiones en plani- } \\
\text { ficación, control, inversiones en seguridad, motivación y re- } \\
\text { ducción de interrupciones. Siendo las dos primeras las más } \\
\text { significativas. }\end{array}$ \\
\hline $\begin{array}{l}\text { Nasirzadeh y } \\
\text { Nojedehib [40] }\end{array}$ & $\begin{array}{l}\text { Estudio de PL en el sector de construcciones median- } \\
\text { te DS. }\end{array}$ & $\begin{array}{l}\text { Resaltan la importancia de la DS en el estudio de la produc- } \\
\text { tividad debido a que permite considerar múltiples factores. }\end{array}$ \\
\hline $\begin{array}{l}\text { Salehi, } \\
\text { Shirouyehzad } \\
\text { \& Dabestani } \\
\text { [41] }\end{array}$ & $\begin{array}{l}\text { Desarrollaron una metodología para la medición de la } \\
\text { PL por medio de la clasificación y estandarización de } \\
\text { los productos. }\end{array}$ & $\begin{array}{l}\text { Usando el NNA - Nearest Neighbour Algorithm- estandariza- } \\
\text { ron las salidas de producción y calcularon el valor de los pro- } \\
\text { ductos, teniendo en cuenta la mano de obra requerida para } \\
\text { la fabricación de las piezas estándar, así como sus procesos } \\
\text { de producción. El modelo validado evidenció crecimiento en } \\
\text { la PL, mejora del sistema de producción, precisión en la pla- } \\
\text { nificación y respuesta a las fluctuaciones del mercado. }\end{array}$ \\
\hline \multicolumn{3}{|c|}{ Enfoque administrativo } \\
\hline $\begin{array}{l}\text { Ríos y Sánchez } \\
\text { [42] }\end{array}$ & $\begin{array}{l}\text { Buscaban identificar mediante herramientas estadísti- } \\
\text { cas indicadores de eficiencia organizacional. }\end{array}$ & $\begin{array}{l}\text { Plantean } 29 \text { criterios como indicadores válidos de efi- } \\
\text { ciencia de una empresa, descritos en su "Cuestionario de } \\
\text { EFO". }\end{array}$ \\
\hline $\begin{array}{l}\text { Cequea, Mon- } \\
\text { roy y Núñez } \\
\text { [43], [44] }\end{array}$ & $\begin{array}{l}\text { Tienen dos investigaciones en el tema. En } 2010 \text { con- } \\
\text { siderando } 45 \text { factores que afectan la productividad, } \\
\text { agrupados en } 3 \text { dimensiones: individual, grupal y orga- } \\
\text { nizacional. } \\
\text { En } 2011 \text { trabajaron el diseño de una escala de medición } \\
\text { de la PL en el sector eléctrico venezolano. }\end{array}$ & $\begin{array}{l}\text { El trabajo de } 2010 \text { corroboró la existencia de una dimen- } \\
\text { sión psicológica, psicosocial y estructural en los factores } \\
\text { evaluados en las dimensiones humanas. } \\
\text { El estudio de } 2011 \text { reveló que las dimensiones asociadas } \\
\text { al factor humano significativas para la PL son: Motivación, } \\
\text { Satisfacción Laboral, Competencias, Participación, Trabajo } \\
\text { en equipo y cohesión, Manejo de conflictos, Formación y } \\
\text { Desarrollo, Cultura organizacional, Liderazgo y Clima orga- } \\
\text { nizacional. }\end{array}$ \\
\hline $\begin{array}{l}\text { Demeter, } \\
\text { Chikán, \& } \\
\text { Matyusz [45] }\end{array}$ & $\begin{array}{l}\text { Analizaron los factores operacionales de cambios en la } \\
\text { PL, considerando las prácticas de trabajo actuales y los } \\
\text { cambios en las prácticas de trabajo a través de progra- } \\
\text { mas de gestión. }\end{array}$ & $\begin{array}{l}\text { Existe un alto grado de correlación entre los cambios de } \\
\text { PL y los cambios en el desempeño organizacional, lo cual } \\
\text { significa que la PL puede ser considerada una fuente rele- } \\
\text { vante del éxito organizacional. }\end{array}$ \\
\hline $\begin{array}{l}\text { Fahed-Sreih } \\
\text { [46] }\end{array}$ & $\begin{array}{l}\text { Examina los factores que contribuyen a la mejora de la } \\
\text { productividad del trabajo, estudiando la relación entre la } \\
\text { planificación del recurso humano, la evaluación formal } \\
\text { de las políticas de contratación, planes de sucesión y la } \\
\text { productividad laboral en diversos sectores del Líbano. }\end{array}$ & $\begin{array}{l}\text { Existe una relación positiva entre la delegación, la deci- } \\
\text { sión mediante empoderamiento, decisiones en conjunto y } \\
\text { el comportamiento de los empleados. Delegación, es la va- } \\
\text { riable más significativa en este estudio. Se demostró que } \\
\text { el liderazgo participativo desempeña un papel esencial en } \\
\text { el comportamiento global del empleado en una organiza- } \\
\text { ción del Líbano. }\end{array}$ \\
\hline $\begin{array}{l}\text { Delmas \& } \\
\text { Pekovic [47] }\end{array}$ & $\begin{array}{l}\text { Analizaron la relación entre las normas ambientales y la } \\
\text { PL, en Francia. }\end{array}$ & $\begin{array}{l}\text { La adopción de normas ambientales aumenta el desem- } \\
\text { peño en el trabajo y puede crear un círculo virtuoso de } \\
\text { interacciones positivas entre la organización y sus emplea- } \\
\text { dos. Los niveles más altos de PL se asocian con mayor } \\
\text { participación financiera, contacto interpersonal, asocia- } \\
\text { ciones entre empresas y prácticas de gestión ambiental } \\
\text { proactiva. }\end{array}$ \\
\hline
\end{tabular}

Fuente: autores.

\section{DISCUSIÓN DE LOS RESULTADOS}

La conceptualización y revisión de estudios de PL desde los enfoques económico, de ingeniería y administrativo resalta la necesidad de una adecuada medición para lograr conclusiones o aportes válidos.

En cuanto a esto, se afirma que la medición de la PL depende de la teoría desde la cual se aborde el concepto de productividad [33], así como de la calidad tanto de los datos de entrada como de las herramientas de procesamiento y análisis.

Frente a estos aspectos, es necesario tener presente las consideraciones para investigar la PL enunciadas por Sargant [48]; quien hizo una revisión de los informes de productividad realizados por el gobierno de EE. UU. por medio de los indicadores de ausentismo y rotación de los empleados, 
llegando a una crítica de la forma como se presentan y analizan los datos de PL, además, resalta que dichos análisis requieren un estándar, y enuncia la falencia que tienen los estudios estadísticos de la época en la simplificación para la obtención de la PL, esto se ve al suponer que los empleados de las empresas son todos iguales, similarmente que al dar por hecho que los tiempos de trabajo a los cuales corresponden los datos de productividad son los mismos.

Aproximadamente un siglo después se continúan presentando dificultades similares en los estudios de PL, asociados con la necesidad de homologación y de rigurosidad en los cálculos, evitando errores en el análisis de información por conglomerados, lo cual implica cuidados en la forma como se agrupa y analiza la información. Lo anterior es importante en estudios de PL desde cualquier enfoque; sin embargo, toma una relevancia especial en el ámbito de la economía, ya que esta se enfoca principalmente en la productividad de las naciones o sectores, en pocas ocasiones estudia individualmente las empresas, lo cual hace que los conglomerados de información sean un aspecto constante en los trabajos de investigación y análisis sobre el tema.

Desde este mismo enfoque de la economía se puede resaltar el reconocimiento de la influencia de la PL en el crecimiento de una economía, "la medida más sencilla de crecimiento es el Producto Interno Bruto (PIB) por habitante, este es el producto de la PL por la fracción de la población que trabaja" [49]. La PL desde la mirada económica se enfoca en su aporte al crecimiento económico, y la relación con variables como el desempleo, la inflación, la educación, entre otras [1], [49].

En la definición de Smith, a quien se le reconoce como pionero en aportar a la conceptualización de la productividad, se resalta el término trabajo, porque se menciona como factor de producción junto con la tierra, y además porque este es realizado por las personas, es decir, desde Smith se puede valorar el impacto de los operarios en la productividad, principalmente debido a la mejora de sus habilidades.

La OIT en su definición resalta la trascendencia de la PL en dos vías, desde un enfoque económico sobre la producción, y desde la mirada socio laboral sobre la calidad de vida.
Frente a los aportes de los investigadores a la PL desde lo económico, se puede concluir sobre el comportamiento de esta y la relación con ciertas variables económicas, elementos que apoyan el planteamiento de políticas de gobierno, o permiten avanzar en teorías que enmarcan los modelos económicos de países y regiones. Sin embargo, las organizaciones encuentran distantes en su ejercicio empresarial los postulados de la PL surgidas en el ámbito económico, principalmente de carácter macro.

En el ámbito de la ingeniería, se observa la optimización de los recursos como un distintivo de la ingeniería, en cuanto a la PL busca el mejor rendimiento de la mano de obra. La ingeniería busca simular y optimizar los recursos organizacionales a través de diferentes métodos, esto representa un aporte para la PL en cuanto al recurso humano, sin embargo, las pymes tienen cierta dificultad para el control de sus recursos, encontrándose en una etapa anterior a la optimización, dadas sus limitaciones, entre ellas las de personal especializado.

La literatura reporta herramientas desde la administración y la ingeniería para la mejora de la productividad, sin embargo, en este documento se desea evidenciar la necesidad de apoyar la gestión de la misma, y específicamente de la PL, proceso donde la medición va a ser uno de los primeros elementos por considerar.

Zuleta y Jaramillo [6] presentan un ejemplo de apoyo en la ingeniería para la mejora de la productividad, manifiestan que las empresas de confecciones, principalmente las de mayor tamaño, deben sus mejoras significativas en productividad de la mano de obra a mejoras electrónicas en los equipos y sobre todo a la capacitación de la mano de obra y las reorganizaciones de los procesos en las plantas. Resaltando el aporte desde la ingeniería industrial asociado a este último elemento.

Es reconocido que desde los planteamientos de Taylor sobre la administración científica se sentaron las bases de la eficiencia, aspecto estrechamente relacionado con la productividad. Recalcando la necesidad de eficiencia y productividad en las organizaciones, a tal nivel de encontrarse referenciadas por diversos autores como objetivos organizacionales [30], [32]. 
Sumanth [50] plantea la administración de la productividad total, la cual define como una "filosofía formal de la administración y un proceso que sigue las cuatro fases del "Ciclo de la Productividad' - medir, evaluar, planear y mejorar-con el fin de incrementar la productividad total y reducir los costos unitarios totales de bienes o servicios, pero con el nivel de calidad más alto posible" ( $p$. 96). Esta definición se enfoca en los objetivos de la gestión de la productividad, comprometiéndose con el control de costos, la mejora de la productividad y la calidad.

\section{CONCLUSIONES}

La productividad laboral es relevante, dado su impacto en las organizaciones, sectores, regiones y comunidad general. El sector confecciones es estratégico para el área metropolitana de Bucaramanga, y las pymes -en mayor grado que las grandes empresas- tienen necesidad de control y mejora de su PL, desde allí se justifica el futuro diseño de un modelo de PL en pymes de confecciones.

La PL es multidimensional, por este motivo el diseño del modelo requiere un estudio desde diferentes puntos de vista, que incluya aspectos: financieros, de gestión, de operaciones y sociales, entre otros. Es decir, considerar las teorías sobre el tema planteadas desde la economía, así como las herramientas y planteamientos sobre la $\mathrm{PL}$ que surgen desde la administración y la ingeniería, e inclusive aportes de otras disciplinas.

Las pymes difieren de las grandes empresas, por este motivo es importante contar con modelos -en este caso particular, de PL- que sean especialmente diseñadas de acuerdo con sus propias características. Esta exploración sobre la PL desde el enfoque de sus tres áreas tradicionales de estudio: la economía, la administración y la ingeniería, permitió identificar importantes aportes en el tema, así como la necesidad de un modelo que los integre y a la vez se adecue a las condiciones particulares de la gestión, el capital y las condiciones en general de las pymes de confecciones en Colombia.

La ingeniería, la economía y la administración buscan la mejora organizacional. En algunas ocasiones análisis de variables similares arrojan resultados opuestos, debido a diferencias en la teo- ría base del estudio, el modo de agregación de las variables o los períodos considerados, entre otros aspectos. Es importante resaltar que la organización es una sola, e independientemente del punto de vista desde el cual se analice los resultados deben brindar conocimiento y orientaciones útiles para el empresario.

El sector de confecciones en el área metropolitana de Bucaramanga mantiene su relevancia, sin embargo, diversos estudios han mostrado como la industria manufacturera, y en particular, las confecciones presentan un bajo nivel de PL. Esto motiva al diseño del modelo para su medición y mejora en pro de estimular una actividad económica de tradición e historia regional y nacional.

La productividad es el determinante primordial de la calidad de vida de un país, de acuerdo con los planteamientos de Porter. De manera que al identificar las variables que explican la PL se abren caminos desde la medición y mejora de la misma a nivel organizacional que lleven a la competitividad empresarial, la mejora de regiones y el aumento de la calidad de vida en general.

Desde el enfoque de Porter, la productividad es la base de la competitividad de allí la importancia de aportar en la gestión de la misma, dando así elementos de apoyo a las pymes para responder a la presión de los mercados globales por ser competitivos, aspecto que se traduce en una necesidad para el sostenimiento organizacional.

Teniendo en cuenta el elemento regional o local de la competitividad (y por ende de la productividad) se hace necesario considerar para el planteamiento del modelo de PL aspectos asociados con el desarrollo regional; en este caso particular si se tienen en cuenta las relaciones de doble vía entre el sector confecciones y la región. Aspecto que lleva a la inclusión en la dinámica del sector confecciones del actor gubernamental, con miras a perfilar el sector como foco para el desarrollo de la región.

Esta revisión de literatura permite conocer aportes valiosos frente a la temática de productividad y PL, así como evidenciar la necesidad de estudios enfocados en las pymes, de manera que sean soporte para la gestión de la PL en estas organizaciones que tienen amplias necesidades, condiciones particulares y son de impacto para la economía y el desarrollo del país. 


\section{AGRADECIMIENTOS}

Un agradecimiento especial al doctor Iván Montoya y la doctora Josefa Ramoni por sus aportes en la estructura de la propuesta de investigación doctoral "Modelo para la productividad laboral de pymes del sector confecciones en el AMB". Este documento es resultado de la revisión inicial de literatura para dicho proyecto de investigación.

\section{REFERENCIAS}

[1] M. E. Porter, Ser competitivo. Deusto, 2009.

[2] Ministerio de Trabajo Empleo y Seguridad Social de Argentina, "La red de RSE y trabajo decente. Un caso de contribución público-privado al desarrollo," Conferencia Europea de RSE, 2010.

[3] C. Chadwick, S. A. Way, G. Kerr, and J. W. Thacker, "Boundary conditions of the High-Investment Human Resource Systems-Small-Firm Labor Productivity Relationship," Pers. Psychol., vol. 66, no. 2, pp. 311-343, Jun. 2013.

[4] M. H. Muñoz Hernández, "Comunicación y productividad en pequeñas y medianas empresas de un cluster textil en Colombia," Contaduría y Administación, vol. 57, pp. 223-244, 2012.

[5] ADN Sectorial, Reporte sectorial, Bucaramanga, 2014.

[6] L. Zuleta and L. Jaramillo, "Reestructuración y competitividad de la cadena textil - confecciones en Colombia," en El crecimiento de la productividad en Colombia, Bogotá: Departamento Nacional de Planeación, 1996, pp. 345-373.

[7] R. Uribe, Costos de la mano de obra en Colombia. Medellín, p. 8, 2011.

[8] Alta Consejería Presidencial para la Competitividad y la Productividad, I. y T. Ministerio de Comercio, Consejo Privado de Competitividad, y Departamento Nacional de Planeación, "Política Nacional de Competitividad y Productividad," Consejo Nacional de Política Económica y Social, Bogotá, p. 87, 2008.

[9] Consejo Privado de Competitividad, "Política de Desarrollo Productivo para Colombia,” Bogotá D.C.: p. 112, 2014.

[10] Consejo Privado de Competitividad, Informe Nacional de Competitividad 2012-2013, Bogotá, 2012.

[11] Institute Management Development, World Competitiveness Ranking 2012, Lausanne, 2012.

[12] World Economic Forum, The Global Competitiveness Report 2012-2013. Geneva: Klaus Schwab, 2012.
[13] Consejo Privado de Competitividad, Informe Nacional de Competitividad 2013-2014, Bogotá, 2013.

[14] M. McMillan and D. Rodrik, "Globalization, structural change and productivity growth," in Making Globalization Socially Sustainable, 2011, pp. 49-85.

[15] Consejo Privado de Competitividad, Informe Nacional de Competitividad 2014-2015, Bogotá D.C., 2014.

[16] J. Atack, F. Bateman, and R. A. Margo, "Steam power, establishment size, and labor productivity growth in nineteenth century American manufacturing," Explor. Econ. Hist., vol. 45, no. 2, pp. 185-198, Apr. 2008.

[17] Cámara de Comercio, Creación de la ventaja competitiva para Colombia. Bogotá, 1994.

[18] L. G. Plata, “Documentos de Interés." Ministerio de Comercio, Industria y Turismo, Bogotá, p. 7, 2009.

[19] Brújula Global S.A.S, “Informe de Gestión 2012 - Programa de Transformación Productiva - PTP," 2013. [Online]. Available: http://www.ptp.com.co/informe_ gestion_2012/. [Accessed: 15-Aug-2014].

[20] E. Enciso Forero, Agenda prospectiva de investigación y desarrollo tecnológico para la cadena productiva de algodón, textil, confecciones en Colombia con énfasis en ropa infantil. Bogotá, 2010.

[21] Inexmoda, Documento Sectorial. Sector textil/confección-diseño y moda en Colombia, Medellín, p. 10, 2012.

[22] Cámara de Comercio de Bucaramanga, "Santander: entorno de negocios competitivos frente al mundo," Centro de Estrategia de Competitividad, UniAndes. Bogotá, p. 225, 2006.

[23] Araújo Ibarra Consultores en Negocios Internacionales, "Quinientos nuevos productos y servicios, para nueve regiones de Colombia, con gran potencial de mercado en Estados Unidos," Resumen ejecutivo, Bogotá, p. 74, 2006.

[24] Corplan, Plan estratégico Bucaramanga Metropolitana 2015. Bucaramanga, p. 146, 2002.

[25] Ministerio de Comercio Industria y Turismo, "Agenda Interna para la Productividad y la Competitividad: Metodología." Consejo Nacional de Política Económica y Social, Bogotá, p. 23, 2004.

[26] Asamblea Departamental de Santander, "Plan de Desarrollo, Santander en serio, el Gobierno de la gente, 2012-2015," Bucaramanga, p. 265, 2012.

[27] A. Smith, The wealth of nations. na, 1776.

[28] Oficina Regional de la OIT para América Latina y el Caribe, "Panorama Laboral 2012. América Latina y el Caribe," Lima, 2012. 
[29] Portal de Productividad Laboral, "Productividad Laboral," Elementos conceptuales, 2014. [Online]. Available: http://www.productividad.org.mx/es/que_es_ portal.aspx. [Accessed: 15-Aug-2014].

[30] P. Drucker, The practice of management. Nueva York, 1954.

[31] G. W. England, "Organizational Goals and Expected Behavior of American Managers," Academy of Management Journal, vol. 10. pp. 107-117, 1967.

[32] R. F. Ericson, "The Impact of Cybernetic Information Technology on Management Value Systems," Management Science, vol. 16. p. B-40,B-60, 1969.

[33] J. Botero García, Los cambios en la productividad: medidas alternativas aplicadas a Colombia" Escuela de Economía y Finanzas, octubre, 2005.

[34] M. Dobija, "Labor productivity vs. minimum wage level,” Mod. Econ., vol. 2, pp. 780-787, 2011.

[35] M. Jedrzejczyk, "Labor productivity parity vs trend of exchange rate," Mod. Econ., vol. 03, no. 06, pp. 780785, Oct. 2012.

[36] D. Lisi, "Analysys of employment protection legislation: a model with endogenous labour productivity," J. Acad. Res. Econ., no. 2, pp. 209-245, 2012.

[37] S. Sultana and Y. Kuroda, "An empirical analysis of labor productivity growth for the taiwanese rice sector," Int. J. Econ. Financ., vol. 4, no. 8, pp. 99-113, 2012.

[38] N. Olimpia, "Labour productivity and human capital in the eu countries: an empirical analys," Ann. Univ. Oradea Econ. Sci., vol. 1, pp. 324-331, 2012.

[39] M. J. Mawdesley and S. Al-Jibouri, “Modelling construction project productivity using systems dynamics approach," Int. J. Product. Perform. Manag., vol. 59, no. 1, pp. 18-36, 2009.

[40] F. Nasirzadeh and P. Nojedehi, "Dynamic modeling of labor productivity in construction projects," Int. J. Proj. Manag., vol. 31, no. 6, pp. 903-911, Aug. 2013.
[41] M. Salehi, H. Shirouyehzad, and R. Dabestani, “Labour productivity measurement through classification and standardisation of products," Int. J. Product. Qual. Manag., vol. 11, no. 1, pp. 57-72, 2013.

[42] M. Fernández-Ríos and J. C. Sánchez, Eficacia organizacional: concepto, desarrollo y evaluación. Ediciones Díaz de Santos, 1997.

[43] M. M. Cequea, C. R. Monroy, and M. A. N. Bottini, "The productivity from a human perspective: Dimensions and factors," Intangible Capital, vol. 7. pp. 549-584, 2011.

[44] M. Cequea and C. Rodríguez-Monroy, "Productividad y factores humanos. Un modelo con ecuaciones estructurales," Interciencia, vol. 37, pp. 121-127, 2012.

[45] K. Demeter, A. Chikán, and Z. Matyusz, "Labour productivity change: Drivers, business impact and macroeconomic moderators," Int. J. Prod. Econ., vol. 131, no. 1, pp. 215-223, May 2011.

[46] J. Fahed-Sreih, "The effect of investing in hiring, human resource planning, and employee development on labor productivity: case of Lebanon.," J. Int. Bus. Res., vol. 11, no. 1, 2012.

[47] M. A. Delmas and S. Pekovic, "Environmental standards and labor productivity: Understanding the mechanisms that sustain sustainability," J. Organ. Behav., vol. 34, pp. 230-252, 2013.

[48] F. Sargant, "The measurement of labor productivity," Q. Publ. Am. Stat. Assoc., vol. 17, no. 131, pp. 289304, 1920.

[49] N. G. Mankiw and E. Rabasco, Principios de economía, 2007.

[50] D. Sumanth, Ingeniería y administración de la productividad. México: McGraw-Hill, 1996. 